Elsevier Editorial System(tm) for Nuclear

Inst. and Methods in Physics Research, B

Manuscript Draft

Manuscript Number: V1799R2

Title: MEASUREMENT OF GAMMA-RAY PRODUCTION CROSS SECTIONS FOR NUCLEAR REACTION $31 \mathrm{P}(\mathrm{p}, \mathrm{PY} 1-0) 31 \mathrm{P}$

Article Type: Full Length Article

Keywords: PIGE, phosphorus, cross section

Corresponding Author: Mr. Hugo Miguel Martins Ferreira da Silva, M.D.

Corresponding Author's Institution: FCT-UNL

First Author: Hugo Miguel Martins Ferreira da Silva, M.D.

Order of Authors: Hugo Miguel Martins Ferreira da Silva, M.D.; Cátia Silva, PhD; Micaela Fonseca; Hélio Luís, PhD; Luís Martins; Vasgo Manteigas; João Cruz; Adelaide P Jesus

Abstract: In order to implement a standard free PIGE analysis of phosphorus, the cross section of the reaction $31 \mathrm{P}(\mathrm{p}, \mathrm{p} \gamma 1-0) 31 \mathrm{P}, \mathrm{Y}_{-}$(1$0)=1266.1 \mathrm{keV}$ was measured for the proton energy range 2-4 MeV, with energy steps ranging from $1 \mathrm{keV}$ on resonances to $10 \mathrm{keV}$ off resonance. This data provides additional information in relation to previous work on this reaction, as a more detailed and extended (in energy) measurement was made. The results obtained in this work were compared within the common energy range with previous measurements. There is a fair agreement within the quoted uncertainties although a larger energy step was used by the other authors and some resonances were missed. Also, a validation was accomplished by introducing the measured excitation function in the ERYA code to calculate thick target phosphorus yields of Ca3(PO4)2, and comparing the calculated values with the corresponding measured yields and also with other thick target yields found in the literature. A good agreement was found. 
We are grateful to the reviewer for his minor comments to the manuscript.

Please see below our response.

1) New references [20,21] were introduced to give a proper reference for the resonances used by the authors to calibrate the accelerator.

2) Antilla --- Anttila: corrected.

3) The correction "Handbook of Ion Beam Analysis" to "Handbook of Modern Ion Beam Materials Analysis" was made. Reference number was updated to number 22 due to the introduction of a new reference before (see point 1). 


\begin{abstract}
In order to implement a standard free PIGE analysis of phosphorus, the cross section of the reaction ${ }^{31} \mathrm{P}\left(\mathrm{p}, \mathrm{p} \gamma_{1-0}\right)^{31} \mathrm{P}, \gamma_{1-0}=1266.1 \mathrm{keV}$ was measured for the proton energy range 2.6-4 MeV, with energy steps ranging from $1 \mathrm{keV}$ on resonances to $10 \mathrm{keV}$ off resonance. This data provides additional information in relation to previous works on this reaction, as a more detailed and extended (in energy) measurement was made. The results obtained in this work were compared within the common energy range with previous measurements. There is a fair agreement within the quoted uncertainties although a larger energy step was used by the other authors and some resonances were missed. Also, a validation was accomplished by introducing the measured excitation function in the ERYA code to calculate thick target phosphorus yields of $\mathrm{Ca}_{3}\left(\mathrm{PO}_{4}\right)_{2}$ and comparing the calculated values with the corresponding measured yields and also with other thick target yields found in the literature. A good agreement was found.
\end{abstract}

Keywords: PIGE, cross section, phosphorus

\title{
MEASUREMENT OF GAMMA-RAY PRODUCTION CROSS SECTIONS FOR NUCLEAR REACTION ${ }^{31} \mathbf{P}\left(\mathbf{p}, \mathbf{p} \gamma_{1-0}\right){ }^{31} \mathbf{P}$
}

\author{
A.P. Jesus ${ }^{\mathrm{a}}$
}

${ }^{\text {a }}$ Laboratório de Instrumentação, Engenharia Biomédica e Física da Radiação

(LIBPhys-UNL), Departamento de Física, Faculdade de Ciências e Tecnologia da Universidade Nova de Lisboa, 2829-516, Monte da Caparica, Portugal

${ }^{\mathrm{b}}$ Universidade Europeia, Laureate International Universities, 1500-210 Lisboa, Portugal

${ }^{c}$ Campus Tecnológico e Nuclear - Instituto Superior Técnico (IST), Estrada Nacional 10, 2695-066 Bobadela, Portugal *email: hugo_miguel_m_silva@hotmail.com 


\section{Introduction}

Proton Induced Gamma-ray Emission, PIGE, is an important technique for light element analysis namely as a complementary technique to Proton Induced X-ray Emission, PIXE. As most of the excitation functions of the pertinent gamma-producing nuclear reactions do not depend smoothly on proton energy, but exhibit narrow and large resonances, till recently most of the PIGE analytical work was done with resource to standards. This method may lead to large uncertainties if the standard is not similar to the sample to be analysed $[1,2,3,4]$. Since the last few years an effort has been made to develop a standard free method based on calculating codes as ERYA [5,6] which integrate the excitation functions in order to obtain the corresponding gamma-ray yields. This effort included the measurement of important cross sections $[7,8,9,10]$ and, concerted by the International Atomic Energy Agency [11], additional measurements and compilation of the data, new and existent in the literature, were produced, being the data available in IBANDL [12].

Phosphorous is an important component of biological samples, namely of bone samples, being a major component of bone mineral structure and an essential element to normal bone homeostasis. For in vivo bone samples, X-ray based techniques are not the best choice to quantify phosphorus as due to the in-depth structural heterogeneity of the samples and the large X-ray self-absorption in the samples, significant geometrical effects are to be expected. Based on gamma-rays with negligible self-absorption, PIGE is certainly a good alternative to deal with in-depth structural heterogeneous samples. From the nuclear reactions of protons on ${ }^{31} \mathrm{P}$, the sole phosphorus isotope, the one with corresponding larger cross sections is the inelastic scattering reaction ${ }^{31} \mathrm{P}\left(\mathrm{p}, \mathrm{p} \gamma_{1-0}\right){ }^{31} \mathrm{P}$, producing a $1266.1 \mathrm{keV}$ gamma-ray line.

For this reaction, there are two excitation functions available in the literature, one of them by Boni et al. (1988) [13], and the other was recently measured by Jokar et al. in 2015 [14]. Boni et al. measured the excitation function for the energy range 2.6-3.8 $\mathrm{MeV}$, in steps of $15-20 \mathrm{keV}$, for the laboratory angle $\theta_{\text {lab }}=90^{\circ}$, and they used a target consisting of $125 \mu \mathrm{g} / \mathrm{cm}^{2} \mathrm{GaP}$ in a Nuclepore filter. Jokar et al. measured the excitation function for the energy range 1.886-3.007 MeV, in steps of $3 \mathrm{keV}$ (on resonances) to 20 $\mathrm{keV}$ (off resonance), for the laboratory angle $\theta_{\text {lab }}=90^{\circ}$ and they used a thin target of $\mathrm{Zn}_{3} \mathrm{P}_{2}$ evaporated on a self-supporting carbon film. These measurements do not provide sufficient information on the reaction ${ }^{31} \mathrm{P}\left(\mathrm{p}, \mathrm{p}^{\prime} \gamma\right){ }^{31} \mathrm{P}$ because: 1$)$ several resonances were missed (particularly in the first publication), due to the large energy step employed in the measurements; 2) in order to improve the sensitivity for phosphorus concentrations lower than $1 \%$, proton energies higher than $3 \mathrm{MeV}$ are needed; 3) since the measured radiation is originated in an excited state with total angular momentum equal to $3 / 2$, the angular distribution of radiation may not be isotropic due to the alignment of that nuclear state; hence, additional measurements at angles other than $90^{\circ}$ are important. 
In this paper we present the results of the measurement of the cross section for the reaction ${ }^{31} \mathrm{P}\left(\mathrm{p}, \mathrm{p} \gamma_{1-0}\right){ }^{31} \mathrm{P}$ in a broader energy range, $2.6-4 \mathrm{MeV}$ for the exit channel $\gamma_{1}=1266.1 \mathrm{keV}$ for the laboratory angle $\theta_{l a b}=130^{\circ}$, in energy steps ranging from 1 $\mathrm{keV}$ (on resonances) to $10 \mathrm{keV}$ (off resonance). Also, in order to validate these measured values, thick target gamma-ray yields were obtained for the $1266 \mathrm{keV}$ line produced by proton bombardment of a $\mathrm{Ca}_{3}\left(\mathrm{PO}_{4}\right)_{2}$ pellet. Yield results given by the ERYA code, using the cross section excitation function as input, were compared with our corresponding experimental values and thick target yields existing in the literature $[15,16,17,18]$.

\section{Experiment}

The analyzed target was prepared by evaporation of a thin film of calcium phosphide on a thin self-supporting silver film. Target characterization was achieved by Rutherford Backscattering Spectrometry (RBS) of a $2000 \mathrm{keV}{ }^{4} \mathrm{He}^{+}$beam produced by a Van de Graaff accelerator (CTN/IST, Lisboa). The spectrum of the RBS analysis is shown in fig. 1. The fit was made with the SIMNRA code [19], considering that the target had two layers, the first one with a thickness of $275 \times 10^{15}$ atoms $/ \mathrm{cm}^{2}$ with $20 \%$ of phosphorous and $80 \%$ of oxygen and the other, a layer of $\mathrm{Ag}$ of thickness $410 \times$ $10^{15}$ atoms $/ \mathrm{cm}^{2}$. The RBS spectrum shows that the amount of $\mathrm{Ca}$ is below what could be expected from the calcium phosphide stoichiometry (due to decomposition during the evaporation stage) and has diffused partially into the Ag film. The target stability was verified by measuring the ratio between phosphorus and silver with a ${ }^{4} \mathrm{He}^{+}$beam before and after the cross section measurements and by repeating measurements at some values of proton energy.

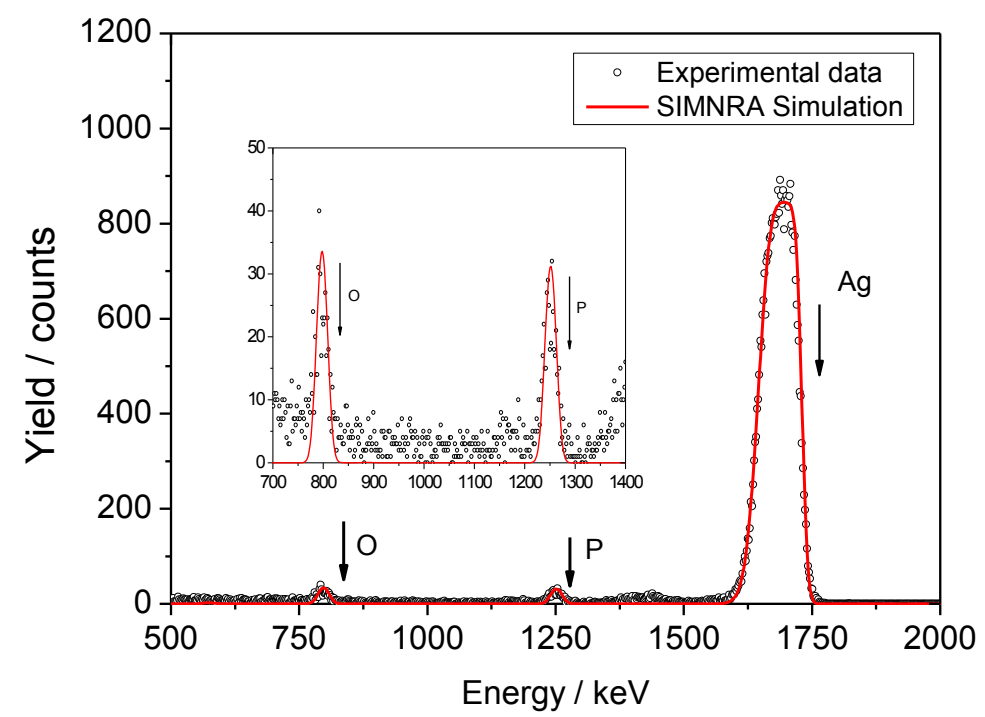


Fig.1 - RBS spectrum of the phosphide target obtained by $\mathrm{Ca}_{3}\left(\mathrm{PO}_{4}\right)_{2}$ evaporation on selfsupporting Ag film together with a SIMNRA fit.

The measurements were carried out at the nuclear reactions tandem accelerator line at CTN/IST. The proton beam was produced by a Duoplasmatron source and accelerated to energies of 2.6-4 MeV by a $3 \mathrm{MV}$ tandem accelerator. The beam current at the target was about $100 \mathrm{nA}$. The proton energy was calibrated by the 1645.1 and 1930.7 resonances of the reaction ${ }^{23} \mathrm{Na}\left(\mathrm{p}, \mathrm{p} \gamma_{1-0}\right)^{23} \mathrm{Na}$ and by the $3470 \mathrm{keV}$ resonance of the ${ }^{16} \mathrm{O}(\mathrm{p}, \mathrm{p}){ }^{16} \mathrm{O}$ reaction $[20,21]$. The reaction chamber was electrically insulated from the beam line working together with the target holder and beam stopper as one Faraday cup for beam charge collection.

Gamma-ray detection was accomplished by a $\mathrm{Ge}(\mathrm{HP})$ detector placed at an angle of $130^{\circ}$ with respect to the beam axis and at a distance of $55.5 \mathrm{~mm}$ from the target. The nominal efficiency and resolution of the detector are $45 \%$ and $1.9 \mathrm{keV}$ for $1.33 \mathrm{MeV}$, respectively. The absolute efficiency was determined by means of calibrated sources ${ }^{133} \mathrm{Ba},{ }^{152} \mathrm{Eu}$, and ${ }^{56} \mathrm{Co}$ placed at the target position. The elastically scattered particles were measured by a PIPS detector with $50 \mathrm{~mm}^{2}$ active area, placed at $155^{\circ}$ to the beam axis and at a distance of $88 \mathrm{~mm}$ from the target, with a depletion layer of $100 \mu \mathrm{m}$ and nominal resolution of $15 \mathrm{keV}$. The particle detector has intrinsic efficiency of $100 \%$ and its solid angle was determined with $2 \%$ uncertainty, by measuring the collimating aperture and the distance between the target and the detector. The solid angle value is $3.65 \times 10^{-3} \mathrm{sr}$.

Figure 2 shows a spectrum of gamma radiation obtained for a proton energy of 3.11 $\mathrm{MeV}$, where the gamma peak pertaining to the ${ }^{31} \mathrm{P}\left(\mathrm{p}, \mathrm{p} \gamma_{1-0}\right){ }^{31} \mathrm{P}$ reaction is clearly seen together with two well known reference gamma rays.

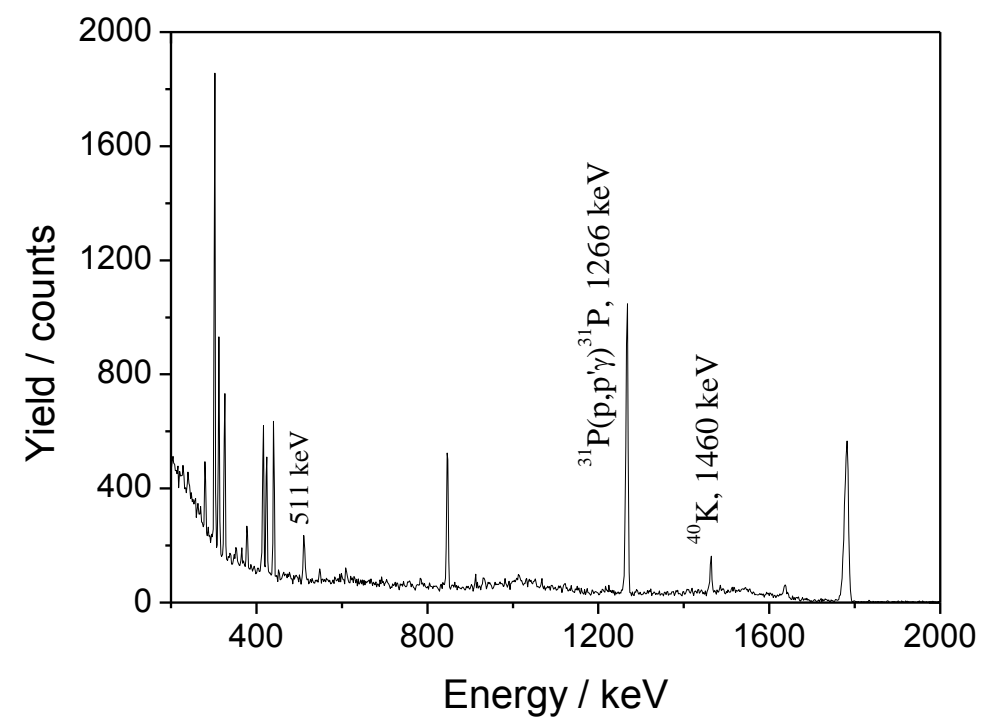


Fig. 2) Gamma-ray spectrum recorded during target irradiation with a proton beam of energy

$3110 \mathrm{keV}$.

In order to avoid the large uncertainties associated to the measurement of the absolute number of incident protons (beam charge collection) and of the target thickness we measured the proton Rutherford backscattering yield of a heavy component of the target (Ag) to obtain the product of the two variables. A spectrum of the scattered protons is given in fig 3, for a $3735 \mathrm{keV}$ proton beam, showing that the Ag peak is well defined and separated from the other peaks.

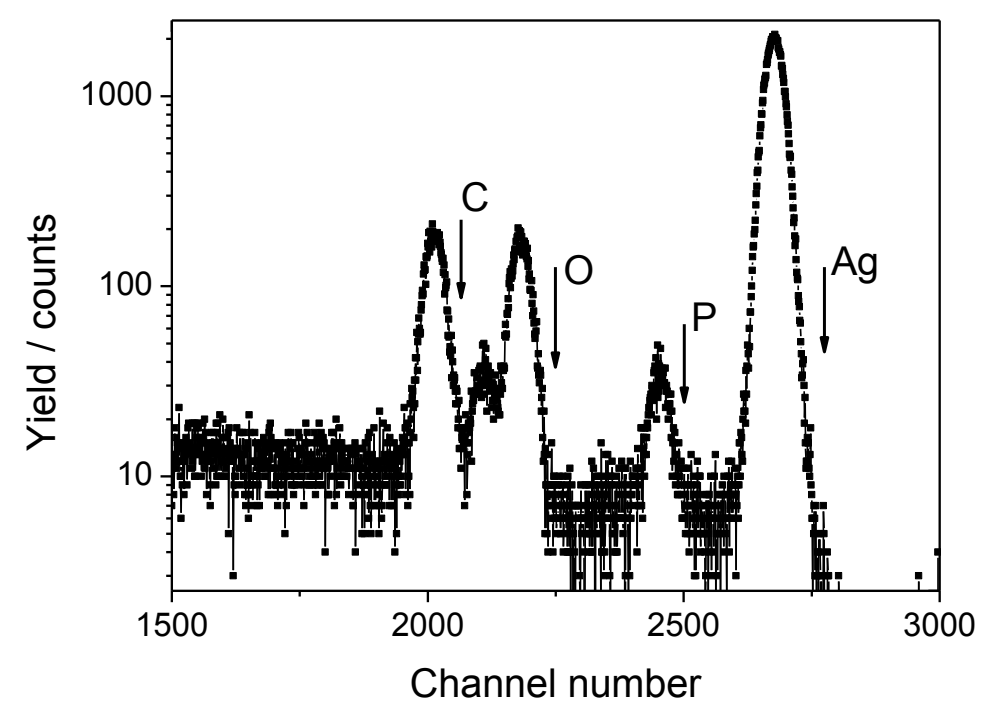

Fig. 3) Spectrum of the protons scattered by the target for $\theta_{\text {lab }}=155^{\circ}$, for $3735 \mathrm{keV} \mathrm{H}^{+}$beam.

The collected charge, for each energy point, was $20 \mu \mathrm{C}$. Dead time correction factors were about $8 \%$ for the gamma detection at the strongest resonances and negligible for particle detection.

The recorded particle spectra allowed an additional monitoring of the accelerator energy. The yield of scattered protons by silver is linearly dependent of the inverse of the square of protons energy, as predicted by the Rutherford cross section.

\section{Results and discussion}

The radiation yield collected within a small solid angle is given by the expression:

$$
Y(E, \theta)=\sigma(E, \theta) N_{b} N_{P} \varepsilon_{a b s}
$$


where $N_{b}$ is the number of incident protons in the beam, $N_{P}$ is the number of phosphorous nuclei per unit area, $\varepsilon_{a b s}$ is the absolute efficiency of the detector and $\sigma(E, \theta)$ is the nuclear reaction cross section at the incident energy $E$ and detection angle $\theta$. This expression is valid for proton energy values off resonance or on resonances that are large when compared with the target energy thickness. In this expression $N_{P}$ may be replaced by $N_{A g} r$, being $r$ the ratio between the number of phosphorous nuclei and silver nuclei taken from the fit showed in figure 1. For this target, this ratio is 0.75 .

The number of protons elastically scattered by silver for a scattering angle $\beta$, and incident energy $E$, within a small solid angle, may be determined by,

$$
Y_{A g}=N_{b} N_{A g} \sigma_{R u t h}(E, \beta) \Omega_{p} \varepsilon_{p}
$$

where $\Omega_{p}$ and $\varepsilon_{p}$ are the solid angle and the efficiency of the particle detector and $\sigma_{R u t h}(E, \beta)$ is the Rutherford cross-section. Using the expressions above the cross section may be given by:

$$
\sigma(E, \theta)=\left[\frac{\sigma_{R u t h}(E, \beta) Y(E, \theta) \Omega_{p} \varepsilon_{p}}{r Y_{A g} \varepsilon_{a b s}}\right]
$$

The cross section as a function of the proton energy was calculated from the measured yields following the last expression and the methods described above. The results have an uncertainty given by a combination of uncertainties of the measured quantities, according to table 1 .

\begin{tabular}{cc}
\hline $\boldsymbol{\gamma}$-ray peak area & $1-3 \%$ \\
\hline p+Ag elastic peak area & $1 \%$ \\
p+Ag Rutherford cross section & $1 \%$ \\
P/Ag atomic ratio & $4 \%$ \\
Particle detector solid angle & $2 \%$ \\
$\boldsymbol{\gamma}$-ray detector efficiency & $6 \%$ \\
Proton beam energy & $0.1 \%$ \\
Overall uncertainty & $8 \%$ \\
\hline
\end{tabular}

Table 1. Uncertainty budget for the measurements.

The excitation function obtained in this work is represented in fig 4 together with the excitation functions measured by Boni et al. [13] and Jokar et al. [14] for comparison purposes. Numerical values of these cross sections are available in IBANDL database [12]. 


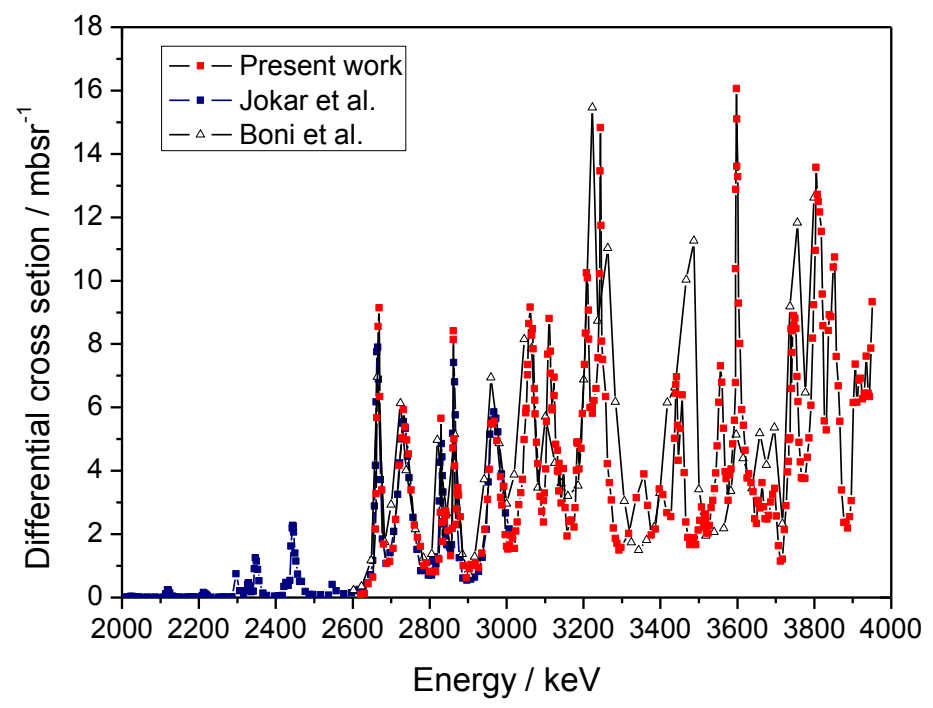

Fig. 4) Excitation function for the inelastic scattering of protons from ${ }^{31} \mathrm{P}$ for the exit channel $\gamma=1266 \mathrm{keV}$ determined experimentally for $\theta_{l a b}=130^{\circ}$ (red points+line). The excitation functions available in the literature (Jokar et al., Boni et al.) are also represented (blue and black points+line).

In the comparison of our results with the other ones, one must bear in mind that the $1266 \mathrm{keV}$ gamma ray is originated in an excited state with total angular momentum equal to $3 / 2$. Hence, the angular distribution of radiation may be anisotropic due to the alignment of that nuclear state. Our results were obtained with the detector positioned at $130^{\circ}$, while for Jokar et al. and Boni et al. the measurements were done at $90^{\circ}$. Nevertheless, in the common covered energy range our results agree with the ones of Jokar et al. within 5\%, with the exception of some resonances present in our work and absent in the one of Jokar et al., probably due to the thicker target they have used. In the relation to the work of Boni et al., there is a clear discrepancy of the energy scale they have used compared to the other two excitation functions. Also, as the energy step is rather large they have missed some resonances and the maximum of other resonances, which hinders the comparison of absolute values of the cross sections.

In order to validate the excitation function obtained for the ${ }^{31} \mathrm{P}\left(\mathrm{p}, \mathrm{p} \gamma_{1-0}\right){ }^{31} \mathrm{P}$ nuclear reaction, we have also measured, for some energy points, the gamma ray yields of a thick target with known composition - a pressed pellet of $\mathrm{Ca}_{3}\left(\mathrm{PO}_{4}\right)_{2}$. The corresponding yields were calculated by the ERYA code [5,6] assuming $15.4 \%$ atomic fraction for phosphorous in the compound, according to the stoichiometry of calcium phosphate. As the excitation function lowest energy point is near $2650 \mathrm{keV}$, the calculated yield cannot take into account the contribution below that energy point. Hence, the experimental yield was assumed for $2650 \mathrm{keV}$ and that value was summed up to the other calculated 
values. Table 2 compares the measured and calculated yields, showing a good agreement between them.

\begin{tabular}{ccc}
\hline $\begin{array}{c}\text { Energy } \\
\text { / keV }\end{array}$ & $\begin{array}{c}\text { Experimental } \\
\text { yields }\end{array}$ & $\begin{array}{c}\text { Calculated } \\
\text { yields }\end{array}$ \\
\hline $\mathbf{2 6 5 0}$ & $1.545 \mathrm{E}+03$ & $1.545 \mathrm{E}+03$ \\
$\mathbf{2 8 0 1}$ & $8.009 \mathrm{E}+03$ & $7.977 \mathrm{E}+03$ \\
$\mathbf{2 9 2 0}$ & $1.143 \mathrm{E}+04$ & $1.133 \mathrm{E}+04$ \\
$\mathbf{3 0 0 7}$ & $1.565 \mathrm{E}+04$ & $1.549 \mathrm{E}+04$ \\
$\mathbf{3 0 9 5}$ & $2.206 \mathrm{E}+04$ & $2.174 \mathrm{E}+04$ \\
$\mathbf{3 1 5 2}$ & $2.698 \mathrm{E}+04$ & $2.646 \mathrm{E}+04$ \\
$\mathbf{3 3 0 0}$ & $3.916 \mathrm{E}+04$ & $3.858 \mathrm{E}+04$ \\
$\mathbf{3 3 6 0}$ & $4.218 \mathrm{E}+04$ & $4.138 \mathrm{E}+04$ \\
$\mathbf{3 4 8 0}$ & $4.806 \mathrm{E}+04$ & $4.836 \mathrm{E}+04$ \\
$\mathbf{3 5 7 5}$ & $5.370 \mathrm{E}+04$ & $5.403 \mathrm{E}+04$ \\
$\mathbf{3 6 4 0}$ & $6.054 \mathrm{E}+04$ & $6.098 \mathrm{E}+04$ \\
\hline $\mathbf{3 7 1 2}$ & $6.373 \mathrm{E}+04$ & $6.462 \mathrm{E}+04$ \\
\hline $\mathbf{3 8 0 4}$ & $7.259 \mathrm{E}+04$ & $7.385 \mathrm{E}+04$ \\
\hline $\mathbf{3 8 8 7}$ & $8.428 \mathrm{E}+04$ & $8.580 \mathrm{E}+04$ \\
\hline
\end{tabular}

Table 2) Experimental and calculated gamma ray yields for a calcium phosphate pellet obtained for our detection system. The yield units are counts/ $\mu \mathrm{C}$.

The experimental yields have only a statistical uncertainty which is less than $1 \%$. In relation to calculated values, these are affected by systematic uncertainties related to the absolute value of the collected charge, to the cross section values and to the stopping powers. As table 1 shows that the agreement between the experimental and calculated values is approximately the same across the whole energy range, one may say that the dependence on energy of the cross section is correct. Also, the absolute values of the cross section are correct within $10 \%$ uncertainty, which is the combined uncertainty of the collected charge and stopping powers.

The detector efficiency does not play a role in this comparison since the same value is used in the cross section determination and yield calculation, canceling out. Nevertheless, it also affects in a systematic way the cross section values. Hence, further validation by comparison with other measured thick target yields is needed. In figure 5 , our results (converted to pure phosphorus yield per steradian) are presented along with previous measurements found in the literature. Also, calculated values using our measured excitation function as input of the ERYA code are shown as a continuous line. 


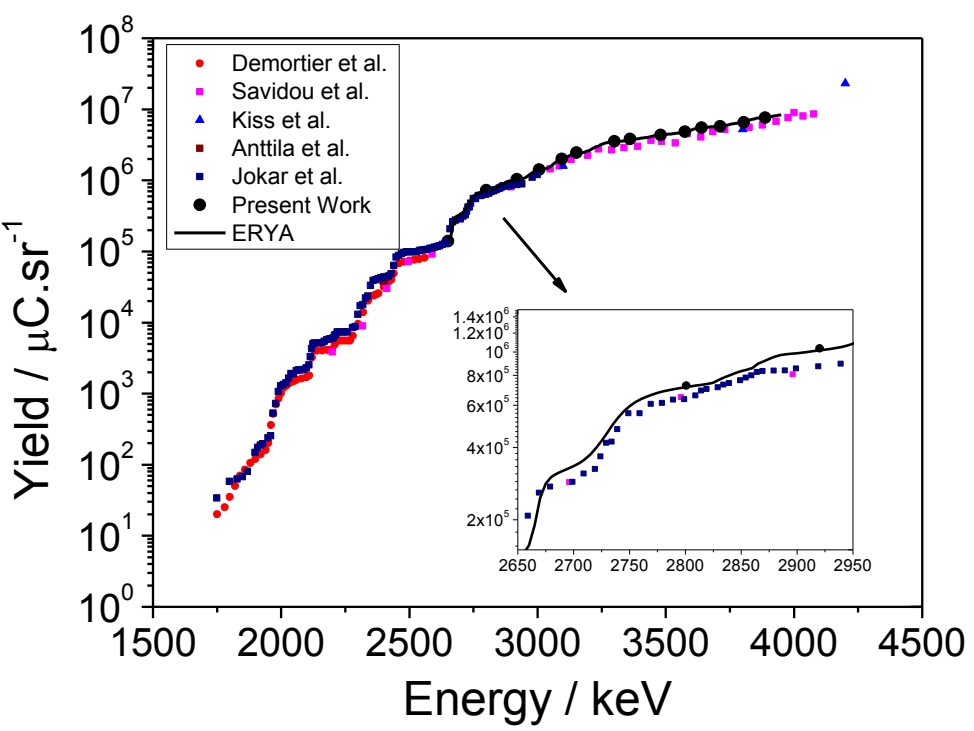

Fig. 5) Thick target yields per steradian corresponding to the $1266 \mathrm{keV}$ gamma-line, emitted by a pure $\mathrm{P}$ target, obtained in the present work and by Demortier et al. [15], Savidou et al. [16], Kiss et al. [17], Anttila et al. [18] and Jokar et al. [14]. The solid line represents calculated values by the ERYA code. [Note that IBANDL provides an erroneous reference to the work represented here of Jokar et al.]

The results of Anttila et al. [18] and Kiss et al. [17] were included in the Handbook of Modern Ion Beam Materials Analysis [22]. These results together with the ones by Demortier et al. [15] and Savidou et al. [16] are now in the IBANDL database.

Previous results of different authors are in good agreement with each other within the uncertainties and our measured values are also in good agreement, being larger than those of Jokar et al and Savidou et al by an average of 14\%, reaching a disagreement with results of Savidou et al of around $22 \%$ for the highest energy values, still within the uncertainties. ERYA calculated values, using the excitation function obtained in this work, follow closely our experimental yields, being consequently also above the results of Jokar et al and Savidou et al, but describing adequately the resonances in the energy range $2650-2950 \mathrm{keV}$, as may be seen in the inset of fig. 5 .

\section{Conclusions}

Values of the cross section for the reaction ${ }^{31} \mathrm{P}\left(\mathrm{p}, \mathrm{p} \gamma_{1-0}\right)^{31} \mathrm{P}, \gamma_{1-0}=1266.1 \mathrm{keV}$ in the proton energy range, $2.6-4 \mathrm{MeV}$ for the laboratory angle $\theta_{\text {lab }}=130^{\circ}$ were obtained. This work represents a more detailed (with energy steps ranging from $1 \mathrm{keV}$ on resonances to $10 \mathrm{keV}$ off resonance) and extended (in proton energy) measurement compared with previous work on the same reaction.

Validation of the corresponding excitation function was accomplished by comparing thick target yield results calculated by the ERYA code using the cross section excitation function 
as input with the corresponding experimental values obtained also in this work for a $\mathrm{Ca}_{3}\left(\mathrm{PO}_{4}\right)_{2}$ pressed pellet. Additional validation came from comparison of our measured thick target yields and ERYA calculated yields with previous measurements found in the literature.

The cross section values obtained in this work were used by the ERYA code to quantify phosphorus in bone tissue (work to be published elsewhere) within a ion beam study of bone diseases. This work shows the importance of the availability of a PIGE method to quantify phosphorus.

\section{Acknowledgments}

H. Silva acknowledges support by grant SFRH/BD/82631/2011 (FCT, Portugal). H. Silva, C. Santos, J. Cruz and A.P. Jesus acknowledge FCT-NOVA. We thank also to the LATR (CTN/IST), where we did our experimental measurements.

\section{References}

[1] M. Fonseca, R. Mateus, C. Santos, J. Cruz, H. Silva, H. Luís, L. Martins, A.P. Jesus, Nuclear Inst. and Methods in Physics Research B 406 (2017) 144-147.

[2] M. Fonseca, H. Luís, N. Franco, M.A. Reis, P.C. Chaves, A. Taborda, J. Cruz, D. Galaviz, N. Fernandes, J.P. Ribeiro, A. P. Jesus, Nucl. Instr. \& Meth. B 269 (2011) 3060-3062.

[3] M. Fonseca, A.P. Jesus, H. Luís, R. Mateus, J. Cruz, L. Gasques, D. Galaviz, J.P. Ribeiro, Nucl. Instr. \& Meth. B 268 (2010) 1806-08.

[4] R. Mateus, M. Fonseca, A.P. Jesus, H. Luís, J.P. Ribeiro, Nuclear Instruments and Methods B 266 (2008) 1490-1492.

[5] R. Mateus, A.P. Jesus, M. Fonseca, H. Luís, J.P. Ribeiro, Nuclear Instruments and Methods B 264 (2007) 340-344.

[6] R. Mateus, M.A. Reis, M. A.; Jesus, J.P. Ribeiro, Nuclear Instruments and Methods in Physics Research Section B 249 (2006) 784-788.

[7] M. Chiari et al., Nuclear Inst. and Methods in Physics Research B (2017) DOI: 10.1016/j.nimb.2017.01.043.

[8] P. Cabanelas, J. Cruz, M. Fonseca, A. Henriques, F. Lourenço, H. Luís, J. Machado, J.P. Ribeiro, A.M. Sánchez-Benítez, P. Teubig, P. Velho, M. Zarza-Moreno, D. Galaviz, A.P. Jesus, Nuclear Inst. and Methods in Physics Research B 381 (2016) 110-113.

[9] P. Dimitriou, H.-W. Becker, I. Bogdanović-Radović, M. Chiari, A. Goncharov, A.P. Jesus, O. Kakuee, Á.Z. Kiss, A. Lagoyannis, J. Räisänen, D. Strivay, A. Zucchiatti, Nuclear Inst. and Methods in Physics Research B 371 (2016) 33-36. 
[10] M. Chiari, B. Melon, B.; Salvestrini, L.; Fonseca, M.; Alves, E.; Jesus, A. P.; Nucl. Instr. \& Meth. B 332 (2014) 355-358.

[11]IAEA-TECDOC-1822,http://wwwpub.iaea.org/books/IAEABooks/12235/Development-of-a-Reference-Database-forParticle-Induced-Gamma-Ray-Emission-PIGE-Spectroscopy

[12] IBANDL database, IAEA, 2017 at http://www-nds.iaea.org/ibandl/.

[13] C. Boni, E. Cereda, G.M. Marcazzan, Nuclear Instr. And Meth. In Physics Research B 35 (1988) 80-86.

[14] A. Jokar, O. Kakuee, M. Lamehi-Rachti, V. Fathollahi, Nuclear Instr. And Meth. In Physics Research B 394 (2017) 28-32.

[15] G. Demortier, F. Bodart, Journal of Radioanalytical Chemistry 12 (1972) 209-219.

[16] A. Savidou, X. Aslanoglou, T. Paradellis, M. Pilakouta, Nuclear Instr. And Meth. In Physics Research B 152 (1999) 12-18.

[17] Á.Z. Kiss, E. Koltay, B. Nyakó, E. Somorjai, A. Anttila, J. Räisänen, Journal of Radioanalytical and Nuclear Chemistry 89/1 (1985) 123-141.

[18] A. Anttila, R. Hänninen,J. Räisänen, Journal of Radioanalytical and Nuclear Chemistry 62 (1981) 193-306;

[19] M. Mayer, AIP Conf. Proc. 475, 541 (1999).

[20] M. Chiari et. al, , Nuclear Instr. And Meth. In Physics Research B 441 (2019) 108118.

[21] D.R. Tilley, H.R. Weller, C.M. Cheves, Nucl. Phys. A 564 (1993) 1.

[22] Y. Wang, M. Nastasi, (eds), Handbook of Modern Ion Beam Materials Analysis, 2nd edition, Materials Research Society, Warrendale, Pennsylvania (2010). 